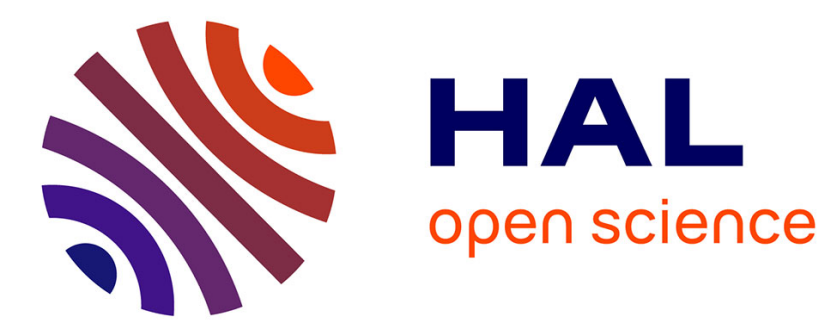

\title{
2-Manifold Tests for 3D Delaunay Triangulation-Based Surface Reconstruction
}

\author{
Maxime Lhuillier
}

\section{To cite this version:}

Maxime Lhuillier. 2-Manifold Tests for 3D Delaunay Triangulation-Based Surface Reconstruction. Journal of Mathematical Imaging and Vision, 2015, 51 (1), pp.98-105. 10.1007/s10851-014-0508-1 . hal-01635381

\section{HAL Id: hal-01635381 https://hal.science/hal-01635381}

Submitted on 27 Nov 2017

HAL is a multi-disciplinary open access archive for the deposit and dissemination of scientific research documents, whether they are published or not. The documents may come from teaching and research institutions in France or abroad, or from public or private research centers.
L'archive ouverte pluridisciplinaire HAL, est destinée au dépôt et à la diffusion de documents scientifiques de niveau recherche, publiés ou non, émanant des établissements d'enseignement et de recherche français ou étrangers, des laboratoires publics ou privés. 


\title{
2-Manifold Tests for 3D Delaunay Triangulation-based Surface Reconstruction
}

\author{
Maxime Lhuillier
}

Received: date / Accepted: date

The reference of this paper is: Maxime Lhuillier, 2Manifold Tests for 3D Delaunay Triangulation-based Surface Reconstruction, Journal of Mathematical Imaging and Vision, 51(1):98-105, 2015

This is the accepted manuscript version that is available at the webpage of the author. The final publication is available at Springer via

http://dx.doi.org/10.1007/s10851-014-0508-1

\begin{abstract}
This is a companion paper of a previous work on the surface reconstruction from a sparse cloud of points, which are estimated by Structure-from-Motion. The surface is a 2-manifold sub-complex of the 3D Delaunay triangulation of the points. It is computed as the boundary of a list of tetrahedra, which grows in the set of Delaunay tetrahedra. Here we detail the proofs for the 2-manifold tests that are used during the growing: we show that the tetrahedron-based test and the test for adding (or subtracting) one tetrahedron to (or from) the list are equivalent to standard tests based on triangles.
\end{abstract}

Keywords Reconstruction - Volumetric models · Geometric topology · Duality and planar graphs

\section{Introduction}

Recently, batch [7] and incremental [11,8] methods have been introduced to reconstruct a surface from sparse Structure-from-Motion points. These methods adaptively divide up the space using a 3D Delaunay triangulation (tetrahedralization) of the points, whose tetrahedra are labeled as free-space or matter using visibility constraints provided by Structure-from-Motion. The surface is then computed as the boundary of a list $O$ of tetrahedra, which grows in the set of free-space tetrahedra. This list must be as large as possible subject to the constraint that the boundary is a 2-manifold.

These methods represent the 3D Delaunay triangulation by the adjacency graph $g$ of the tetrahedra, since this is adequate for both memory space [1] and tetrahedron labeling. The surface is implicitly represented by the set which includes every $g$ edge linking a tetrahedron in $O$ and another one not in $O$. However, the standard 2-manifold tests for a surface are defined on a
Maxime Lhuillier

Institut Pascal, 63171 Aubière cedex, France

Tel.: +33 (0) 473407593

Fax: $+33(0) 473407262$

E-mail: maxime.lhuillier@univ-bpclermont.fr 
surface-based representation (e.g. the adjacency graph of triangles), not a volumetric one as in ours.

Thus, two 2-manifold tests directly based on tetrahedra are used in [7] for efficiency. The former checks that the surface is a 2-manifold near surface vertex $\mathbf{v}$ by counting the connected components of a $g$ sub-graph of the tetrahedra having $\mathbf{v}$ as vertex. The latter checks that a single tetrahedron can be added to $O$ without loss of the 2-manifold property.

However, the complete proofs of the two tests have not been published before. The former has a short and sketch proof in Appendix A of [7], which ignores the case of a surface with a vertex at the boundary of the Delaunay triangulation. The latter only has a visual proof [2] (a figure) or a partial proof [4] (sufficient but not necessary condition) in the special case where the surface encloses all input points. Here we give detailed proofs with necessary and sufficient conditions in all cases. The paper provides prerequisites (Sec. 2), statements (Sec. 3) and proofs (Sec. 5 and 4) of the tests.

\section{Prerequisites (Main Definitions, Properties)}

The majority of prerequisites are in $[6,5]$. We introduce integers $k \geq 0$ and $n>0$. A (geometric) simplex $\sigma$ is the convex hull of $k+1$ points $\mathbf{v}_{0} \cdots \mathbf{v}_{k}$ in general position in $\mathbb{R}^{n}$, i.e. $\mathbf{v}_{1}-\mathbf{v}_{0} \cdots \mathbf{v}_{k}-\mathbf{v}_{0}$ are linearly independent. We say that $\sigma^{\prime}$ is a face of $\sigma$ if $\sigma^{\prime}$ is the convex hull of some of the $\mathbf{v}_{i}$ above (thus $\sigma^{\prime} \subseteq \sigma$ ). A simplicial complex $K$ in $\mathbb{R}^{n}$ is a finite set of simplices in $\mathbb{R}^{n}$ such that

1. if $\sigma \in K$ and $\sigma^{\prime}$ is a face of $\sigma, \sigma^{\prime} \in K$.

2. if $\left\{\sigma, \sigma^{\prime}\right\} \subseteq K, \sigma \cap \sigma^{\prime}$ is empty or a face of $\sigma$ and $\sigma^{\prime}$.

Let $\mathcal{V}^{\prime}$ be a set. An abstract simplicial complex $\mathcal{S}$ is a finite set of subsets of $\mathcal{V}^{\prime}$ such that $A \in \mathcal{S}$ and $B \subset A$ imply $B \in \mathcal{S}$. It is implicitly defined by every simplicial complex $K$ as follows: $\mathcal{V}^{\prime}$ is the vertex set of $K$ and $\mathcal{S}$ is the family of the vertex sets of the $K$ simplices. Conversely, we say that $K$ is a realization of $\mathcal{S}$ in $\mathbb{R}^{n}$. In this paper, we often use the same notation for a simplicial complex and its abstract version.

The elements of $\mathcal{S}$ are called (abstract) simplices. Their faces are their subsets. If simplex $\sigma$ has exactly $k+1$ vertices, $\sigma$ has a dimension $k$. Simplex $\sigma$ is a vertex, edge, triangle or tetrahedron if $k$ is $0,1,2$ or 3 , respectively. We use bold fonts for vertices in an (abstract) simplicial complex, e.g. a is a vertex, ab is an edge, $\mathbf{a b c}$ is a triangle. We say that edge $\mathbf{a b}$ is $\mathbf{c}$-opposite in triangle abc, triangle abc is $\mathbf{d}$-opposite in tetrahedron abcd, $\sigma$ is $\sigma^{\prime}$-incident if $\sigma^{\prime} \subset \sigma$.

Let $L^{\prime}$ be a subset of simplices in an (abstract) simplicial complex. The closure $c\left(L^{\prime}\right)$ is the set of the faces of the $L^{\prime}$ simplices; $c\left(L^{\prime}\right)$ is an (abstract) simplicial complex which includes $L^{\prime}$. Let $\sigma \in L^{\prime}$. If $\sigma$ is not included in another simplex in $L^{\prime}$, we say that $\sigma$ is maximal in $L^{\prime}$. In a $k$-dimensional pure (abstract) simplicial complex, every maximal simplex has dimension $k$, therefore every simplex is included in a simplex of dimension $k$.

The boundary $\partial \sigma$ of a $k$-dimensional simplex $\sigma$ is the set of its $k+1$ faces of dimension $k-1$ : a tetrahedron has four triangles, a triangle has three edges, an edge has two vertices, a vertex has an empty boundary. If all simplices in $L^{\prime}$ are tetrahedra, boundary $\partial L^{\prime}$ is the set of triangles such that every triangle is a face of exactly one tetrahedron in $L^{\prime}$. In this case, $c\left(L^{\prime}\right)$ and $c\left(\partial L^{\prime}\right)$ are $3 \mathrm{D}$ and $2 \mathrm{D}$ pure (abstract) simplicial complexes, respectively.

Let $\mathcal{V}$ be a set of $m \geq 4$ points in $\mathbb{R}^{3}$. Let $T$ be a set of tetrahedra which meets three conditions:

1. $\mathcal{V}$ is the vertex set of the tetrahedra in $T$.

2. the convex hull of $\mathcal{V}$ is the union of tetrahedra in $T$.

3. the circumscribing sphere of every tetrahedron in $T$ does not contain a $V$ vertex in its interior.

Thus $c(T)$ is a $3 \mathrm{D}$ pure simplicial complex in $\mathbb{R}^{3}$, which is called a 3D Delaunay triangulation of $V$. Every triangle in $c(T)$ is included in exactly two tetrahedra in $c(T)$, except those in $\partial T$ (we have $\partial T \neq \emptyset$ ).

To avoid special cases in the statements and proofs of our tests, we extend $c(T)$ to a 3D pure abstract simplicial complex where every triangle is included in exactly two tetrahedra [1]. Its vertex set is $\mathcal{V}^{\infty}=\mathcal{V} \cup$ $\left\{\mathbf{v}_{\infty}\right\}$, where new vertex $\mathbf{v}_{\infty}$ is called the infinite vertex $\left(\mathbf{v}_{\infty} \notin \mathbb{R}^{3}\right)$. Its tetrahedron set is $T^{\infty}=T \cup\left\{\mathbf{a b c v}_{\infty}\right.$, abc $\in \partial T\}$ (here we use the abstract versions of $c(T)$ simplices). Thus $c\left(T^{\infty}\right)$ is a $3 \mathrm{D}$ pure abstract simplicial complex including the abstract version of $c(T)$. Assume $\sigma \in c\left(T^{\infty}\right)$. We say that $\sigma$ is infinite if $\mathbf{v}_{\infty}$ is a vertex of $\sigma$, otherwise $\sigma$ is finite.

Let $k \in\{2,3\}$ and $L$ be a subset of $k$-dimensional simplices in $c\left(T^{\infty}\right)$. If $k=3$, we use notation $L^{c}=T^{\infty} \backslash$ $L$. Let $\tau$ be a vertex or an edge in $c\left(T^{\infty}\right)$. We also use notation $L_{\tau}=\{\sigma \in L, \tau \subset \sigma\}$. For example, $T_{\tau}^{\infty}$ is the set of the $\tau$-incident tetrahedra in $T^{\infty}, L_{\tau}^{c}$ is the set of the $\tau$-incident tetrahedra in $T^{\infty} \backslash L$. If $\tau$ is the edge with vertices $\mathbf{a}$ and $\mathbf{b}, L_{\tau}=L_{\mathbf{a}} \cap L_{\mathbf{b}}$. Indeed, a triangle (or a tetrahedron) includes edge $\mathbf{a b}$ iff it includes vertices a and b ("iff" means "if and only if"). Assuming vertex $\mathbf{v} \in c(T), \mathbf{v} \in c(\partial T)$ iff $T_{\mathbf{v}}^{\infty}$ contains at least one infinite tetrahedron. We also have $\partial T_{\mathbf{v}}^{\infty}=\left\{\mathbf{a b c}, \mathbf{a b c v} \in T^{\infty}\right\}$.

A cycle is a graph with $m$ vertices $\mathbf{q}_{1} \cdots \mathbf{q}_{m}$ and $m$ edges $\mathbf{q}_{1} \mathbf{q}_{2}, \cdots, \mathbf{q}_{m-1} \mathbf{q}_{m}, \mathbf{q}_{m} \mathbf{q}_{1}$ where $m>2$. Let $(V, E)$ be the graph with vertex set $V$ and edge set $E$. Let $\left\{V_{1}, V_{2}\right\}$ be a partition of $V$. Let $F$ be the set of all edges in $E$ such that every edge in $F$ has a vertex in 
$V_{1}$ and a vertex in $V_{2}$. Set $F$ is a cut in $(V, E)$. Graph $(V, E \backslash F)$ has at least two connected components: one in $V_{1}$ and another one in $V_{2}$. Set $F$ is a minimal cut [3] if $V_{1}$ and $V_{2}$ are two connected components in $(V, E \backslash F)$.

Two simplices $\sigma_{1}$ and $\sigma_{2}$ in $L$ are adjacent if $\sigma_{1} \cap \sigma_{2}$ has dimension $k-1$ (e.g. two tetrahedra are adjacent if their intersection is a triangle). Let $g_{L}$ be the adjacency graph of $L$ : the vertices of $g_{L}$ are the simplices in $L$, every edge of $g_{L}$ links two distinct vertices in $g_{L}$ if the two corresponding simplices in $L$ are adjacent. Let $\tau$ be a vertex or and edge in $c(T)$, and $g$ be the adjacency graph of $T^{\infty}$. The adjacency graph of $T_{\tau}^{\infty}$ is shortened $g_{\tau} ; g_{\tau}$ is a connected sub-graph of $g$. If $\tau$ is an edge, $g_{\tau}$ is a cycle. If vertex $\mathbf{v}$ is in $c(T)$, the vertices and edges of $c\left(\partial T_{\mathbf{v}}^{\infty}\right)$ form a connected graph. Proofs for these properties are in the supplementary material (available at http://maxime. Ihuillier.free.fr/pJmiv15Supp.pdf).

A $n$-ball $B$ has center $\mathbf{x} \in \mathbb{R}^{n}$ and radius $r>0$, i.e. $B=\left\{\mathbf{y} \in \mathbb{R}^{n},\|\mathbf{x}-\mathbf{y}\|<r\right\}$ where $\|$.$\| is the Euclidean$ norm. $B$ is open in $\mathbb{R}^{n}$. If $L \subseteq c(T)$, we define $|L|=$ $\cup_{\sigma \in L} \sigma$ (union of convex hulls). A set included in $\mathbb{R}^{n}$ (e.g. $|L|)$ has the topology induced by the $n$-balls [12]. Topological spaces $X$ and $Y$ are homeomorphic if there is a bijective and continuous function $\varphi$ such that $\varphi^{-1}$ is continuous and $\varphi(X)=Y ; \varphi$ is a homeomorphism.

Let $M \subseteq \mathbb{R}^{3}$. We say that $\mathbf{x} \in M$ is regular in $M$ if x has a neighborhood in $M$ homeomorphic to a 2-ball. Intuitively, $M$ has a local $2 \mathrm{D}$ parametrization at $\mathbf{x}$. If every $\mathbf{x} \in M$ is regular in $M, M$ is a 2-manifold in $\mathbb{R}^{3}$.

\section{Overview of 2-Manifold Tests}

Let $O$ be a list of tetrahedra included in $T^{\infty}$ such that every triangle in $\partial O$ is finite. We would then like to check that $|\partial O|$ is a 2-manifold. We use two definitions.

Definition 1 (Good Edges) An edge in $c(\partial O)$ is $\partial O$ good if it is included in exactly two triangles of $\partial O$.

Definition 2 (Good Vertex) A vertex $\mathbf{v}$ in $c(\partial O)$ is $\partial O$-good if the $\mathbf{v}$-incident triangles in $\partial O$ can be ordered as $t_{0}, t_{1}, \cdots t_{k}$ such that $t_{i} \cap t_{(i+1) \bmod (k+1)}$ is an edge for every $i \in\{0,1, \cdots k\}$.

According to [10],

Theorem 1 (Global Test) $|\partial O|$ is a 2-manifold iff the vertices and the edges in $c(\partial O)$ are $\partial O$-good.

We have the same test for a single vertex:

Theorem 2 (Triangle-based Test) A vertex $\mathbf{v}$ in $c(\partial O)$ is regular in $|\partial O|$ iff $\mathbf{v}$ and the $\mathbf{v}$-incident edges in $c(\partial O)$ are $\partial O$-good.
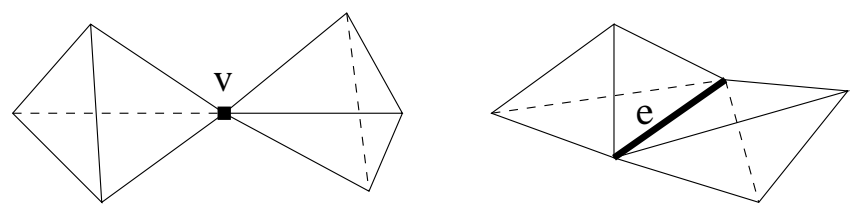

Fig. 1 Two examples where $|\partial O|$ is non manifold. $O$ has two tetrahedra, whose intersection is vertex $\mathbf{v}$ or edge $e$.

For the completeness of the paper, we also provide proofs of these theorems in the supplementary material. Fig. 1 shows two examples where $O$ has two tetrahedra and both conditions (good vertices and good edges) are needed to obtain a 2-manifold. On the left, vertex $\mathbf{v}$ is not regular in $|\partial O|$ since $\mathbf{v}$ is not $\partial O$-good. On the right, every point of edge $e$ is not regular in $|\partial O|$ since $e$ is not $\partial O$-good. All other vertices and edges are $\partial O$-good.

Since Theorem 1 condition is the conjunction of the conditions of Theorem 2 for all vertices in $c(\partial O)$,

Corollary $1|\partial O|$ is a 2-manifold iff every vertex of $c(\partial O)$ is regular in $|\partial O|$.

The Triangle-based Test can be rewritten using edges [2]:

Corollary 2 (Edge-based Test) A vertex $\mathbf{v}$ in $c(\partial O)$ is regular in $|\partial O|$ iff the $\mathbf{v}$-opposite edges in the triangles of $\partial O$ having $\mathbf{v}$ as vertex form a cycle.

Now assume that our data structure is the adjacency graph $g$ of the tetrahedra of $T^{\infty}$. Boundary $\partial O$ is represented by a cut: the $g$ edges between $O$ and $O^{c}$. In this case, the following test is preferred [7] over the Triangle-based Test.

Theorem 3 (Tetrahedron-based Test) Let $g_{\mathbf{v}}^{O}$ be the graph obtained from $g_{\mathbf{v}}$ by removing the edges between a tetrahedron in $O$ and another in $O^{c}$. Vertex $\mathbf{v} \in c(\partial O)$ is regular in $|\partial O|$ iff $g_{\mathbf{v}}^{O}$ exactly has 2 connected components. These components are $O_{\mathbf{v}}$ and $O_{\mathbf{v}}^{c}$.

The proof is in Sec. 5. In $[8,7]$, the implementation of this test is a graph traversal of $g_{\mathbf{v}}^{O}$.

Fig. 2 shows examples to experiment the above tests:

1. $\mathbf{v} \notin c(\partial O), g_{\mathbf{v}}^{O}$ is connected since $g_{\mathbf{v}}^{O}=g_{\mathbf{v}}$.

2. $\mathbf{v}$ is not regular in $|\partial O|$, the $\mathbf{v}$-opposite edges does not form a cycle, $g_{\mathbf{v}}^{O}$ has 3 connected components.

3. $\mathbf{v}$ is regular in $|\partial O|$, the $\mathbf{v}$-opposite edges form a cycle, $g_{\mathbf{v}}^{O}$ has exactly 2 connected components.

If we would like to add a single tetrahedron in $O$, a specific test is given in Appendix of [11]:

Theorem 4 (Adding One Tetrahedron) Assume that $|\partial O|$ is a 2-manifold. Let $\Delta \in T \backslash O$ and $f$ be the number of triangles in $\partial \Delta \cap \partial O$. Thus $|\partial(O \cup\{\Delta\})|$ is a 2-manifold iff one of the following conditions is meet: 


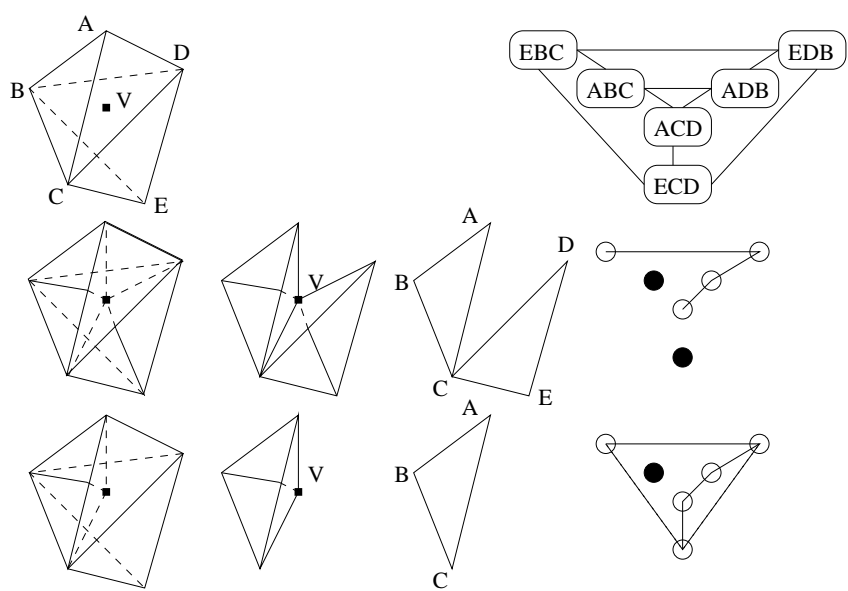

Fig. 2 The Triangle/Edge/Tetrahedron-based tests in three cases (top, middle, bottom) such that $T_{\mathbf{v}}^{\infty}$ has 6 tetrahedra. Top: $\mathbf{v} \notin c(\partial O)$ and $O_{\mathbf{v}}=T_{\mathbf{v}}^{\infty}$. Middle: $\mathbf{v}$ is non regular and $O_{\mathbf{v}}=T_{\mathbf{v}}^{\infty} \backslash\{\mathbf{a b c v}, \mathbf{c d e v}\}$. Bottom: $\mathbf{v}$ is regular and $O_{\mathbf{v}}=$ $T_{\mathbf{v}}^{\infty} \backslash\{\mathbf{a b c v}\}$. Left: tetrahedra in $O_{\mathbf{v}}$. Middle-left: $\mathbf{v}$-incident triangles in $\partial O$. Middle-right: graph of the $\mathbf{v}$-opposite edges in triangles of $\partial O$. Right: graph $g_{\mathrm{v}}^{O}$ (a circle is filled iff the corresponding tetrahedron is in $O_{\mathrm{v}}^{c}$ ).

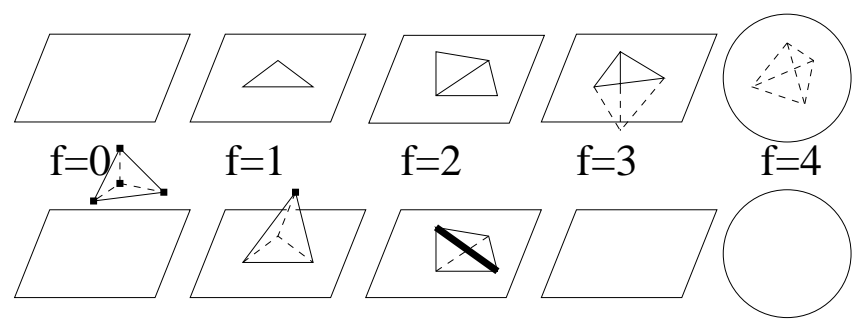

Fig. 3 Cases $f \in\{0,1,2,3,4\}$ where tetrahedron $\Delta$ can be added to $O$ such that $|\partial O|$ is maintained 2-manifold. Top: triangles of $\partial O \cap \partial \Delta$ before adding $\Delta$ to $O(O$ is below the plane, except on the right where $|O|$ is a ball with a tetrahedral cavity). Bottom: surface $|\partial(O \cup\{\Delta\})|$. The bold vertices and edges should not be included in a tetrahedron of $O$.

- if $f=0$ and every $\Delta$ vertex $\mathbf{v}$ meets $O_{\mathbf{v}}=\emptyset$.

- if $f=1$ and the $\Delta$ vertex $\mathbf{v}$, which is opposite to the $\Delta$ triangle included in $\partial O$, meets $O_{\mathbf{v}}=\emptyset$.

- if $f=2$ and the $\Delta$ edge $e$, which is not in one of the two $\Delta$ triangles included in $\partial O$, meets $O_{e}=\emptyset$.

- if $f=3$.

- if $f=4$.

Fig. 3 shows the five cases. The proof is in Sec. 4. In [11, $7,8]$, we check that $O_{\mathbf{v}}=\emptyset$ or $O_{e}=\emptyset$ by a single reading of $T_{\mathbf{v}}^{\infty}$, the set of $\mathbf{v}$-incident tetrahedra (for edge $e$ with vertices $\mathbf{v}$ and $\mathbf{w}$, we look for the tetrahedra in $T_{\mathbf{v}}^{\infty}$ having vertex $\mathbf{w})$.

Since $\partial O=\partial O^{c}$ and subtracting one tetrahedron from $O$ is like adding one tetrahedron to $O^{c}$, we can rewrite the addition Theorem 4 as a subtraction one. This result is used in [8] (without proof). A similar result is also used in $[2,4]$ where only cases $f \in\{1,2\}$ occur.

\section{Proof for "Adding One Tetrahedron"}

First we study the simplicial complex "between $O$ and $\Delta "$ in the two following lemmas. Intuitively, it is indifferently defined by the intersection of closures of $O$ and $\Delta$, or by the intersection of closures of $\partial O$ and $\partial \Delta$. We have $O \subset T^{\infty}, \partial O \subset c(T)$ and $\Delta \in T \backslash O$ in the paper.

Lemma $1 c(O) \cap c(\Delta)$ is a simplicial complex in $\mathbb{R}^{3}$. Furthermore, $c(O) \cap c(\Delta)=c(\partial O) \cap c(\partial \Delta)$. As a consequence, the triangles in $c(O) \cap c(\Delta)$ are exactly those in $\partial \Delta \cap \partial O$.

Lemma $2|\partial \Delta| \cap|\partial O|=|c(\partial O) \cap c(\partial \Delta)|$. If $c(O) \cap c(\Delta)$ is $2 D$ pure, $|\partial \Delta| \cap|\partial O|=|\partial \Delta \cap \partial O|$.

We also examine the triangles in $\partial(O \cup\{\Delta\})$ :

Lemma $3 \partial(O \cup\{\Delta\})=(\partial O \cup \partial \Delta) \backslash(\partial O \cap \partial \Delta)$.

The proofs of the three lemmas above are in Appendix A. They can be omitted at the first reading since they are essentially consequences of basic properties of (abstract) simplicial complexes.

In Lemma 4, we convert the condition of Theorem 4 to a more tractable condition for our proof. It is also useful to visualize all cases in Theorem 4 at once.

Lemma 4 The condition of Theorem 4 is meet iff $c(O) \cap$ $c(\Delta)$ is a $2 D$ pure simplicial complex.

Proof If $f \in\{0,1,2\}$, we show that the conditions of Theorem 4 are not meet iff there exists a maximal simplex in $c(O) \cap c(\Delta)$ which is not a triangle.

Assume $f=0$. According to Lemma $1, c(O) \cap c(\Delta)$ does not contain triangles. If there is a vertex $\mathbf{v}$ of $\Delta$ such that $O_{\mathbf{v}} \neq \emptyset, \mathbf{v} \in c(O) \cap c(\Delta)$. There is therefore a maximal simplex $\tau$ in $c(O) \cap c(\Delta)$ which contains $\mathbf{v}$, and $\tau$ is not a triangle. Conversely, let $\tau$ be a maximal simplex in $c(O) \cap c(\Delta)$ (which is not a triangle). Every vertex $\mathbf{v}$ of $\tau$ is in simplicial complex $c(O) \cap c(\Delta)$. This implies that $\mathbf{v}$ is a vertex of $\Delta$ and $O_{\mathbf{v}} \neq \emptyset$.

Assume $f=1$. We have $\Delta=\mathbf{a b c v}$ and $\partial O \cap \partial \Delta=$ $\{\mathbf{a b c}\}$. According to Lemma 1 , abc is the unique triangle in $c(O) \cap c(\Delta)$. If $O_{\mathbf{v}} \neq \emptyset, \mathbf{v} \in c(O) \cap c(\Delta)$. There is a maximal simplex $\tau$ in $c(O) \cap c(\Delta)$ which contains $\mathbf{v}$, and $\tau$ is not a triangle (otherwise $\tau=\mathbf{a b c}$, which is impossible). Conversely, let $\tau$ be a maximal simplex in $c(O) \cap c(\Delta)$ which is not a triangle. Assume (reductio ad absurdum) that $\mathbf{v}$ is not a vertex of $\tau$. Since $\tau \subset \mathbf{a b c v}$, $\tau \subseteq$ abc. Since $\tau$ is maximal, $\tau=$ abc (impossible). Thus $\mathbf{v} \in c(O) \cap c(\Delta)$, and we obtain $O_{\mathbf{v}} \neq \emptyset$.

Assume $f=2$. We have $\Delta=$ abcd, $\partial O \cap \partial \Delta=$ $\{\mathbf{a b c}, \mathbf{b c d}\}$ and $e=\mathbf{a d}$. According to Lemma 1, the triangles in $c(O) \cap c(\Delta)$ are abc and bcd. If $O_{e} \neq \emptyset$, $e \in c(O) \cap c(\Delta)$. Since $c(O) \cap c(\Delta)$ only has two triangles 
abc and bcd, edge $e$ is maximal. Conversely, let $\tau$ be a maximal simplex in $c(O) \cap c(\Delta)$ which is not a triangle. Since every $\Delta$ vertex is in abc or bcd, $\tau$ is an edge. Since every $\Delta$ edge except $e$ is included in abc or bcd, $\tau=e$. Thus $O_{e} \neq \emptyset$.

Assume $f=3$ or $f=4$. Thus $c(O) \cap c(\Delta)$ contains $f$ triangles of $\Delta$ (Lemma 1$)$, and every vertex and edge of $\Delta$ is in these triangles. So $c(O) \cap c(\Delta)$ is $2 \mathrm{D}$ pure.

Last we show that Lemma 4 condition is necessary (in Lemma 5) and sufficient (in Lemma 6) to conclude that $|\partial(O \cup\{\Delta\})|$ is a 2-manifold. We obtain Theorem 4.

Lemma 5 If $c(O) \cap c(\Delta)$ is not a $2 D$ pure simplicial complex, $|\partial(O \cup\{\Delta\})|$ is not a 2-manifold.

Proof The principle of the proof is the following. Since $c(O) \cap c(\Delta)$ is not 2D pure, it contains a maximal vertex or edge $\tau$. Then we show that $\tau$ is not $\partial(O \cup\{\Delta\})$-good by studying $(\partial(O \cup\{\Delta\}))_{\tau}$, the triangles in $\partial(O \cup\{\Delta\})$ which include $\tau$. Lastly we use Theorem 1: $|\partial(O \cup\{\Delta\})|$ is not a 2-manifold since $\tau$ is not $\partial(O \cup\{\Delta\})$-good.

First we detail $(\partial(O \cup\{\Delta\}))_{\tau}$ thanks to Lemma 3 and $(\partial O \cup \partial \Delta) \backslash(\partial O \cap \partial \Delta)=(\partial O \backslash \partial \Delta) \cup(\partial \Delta \backslash \partial O)$. Using shortened notations $L_{\tau}^{O}=(\partial O \backslash \partial \Delta)_{\tau}$ and $L_{\tau}^{\Delta}=$ $(\partial \Delta \backslash \partial O)_{\tau}$, we have $(\partial(O \cup\{\Delta\}))_{\tau}=L_{\tau}^{O} \cup L_{\tau}^{\Delta}$.

Assume that $\tau$ is a vertex. Since $\tau \in c(\partial O) \cap c(\partial \Delta)$ (Lemma 1), there are triangles $t_{O}$ and $t_{\Delta}$ such that $\tau \subset t_{O} \in \partial O$ and $\tau \subset t_{\Delta} \in \partial \Delta$. Furthermore $t_{O} \notin \partial \Delta$ (otherwise, $\tau \subset t_{O} \in \partial O \cap \partial \Delta \subseteq c(O) \cap c(\Delta)$ and thus $\tau$ is not maximal) and similarly $t_{\Delta} \notin \partial O$. We obtain $L_{\tau}^{\Delta} \neq \emptyset$ and $L_{\tau}^{O} \neq \emptyset$. Let $t_{\Delta}^{\prime} \in L_{\tau}^{\Delta}, t_{O}^{\prime} \in L_{\tau}^{O}$ and $e=t_{O}^{\prime} \cap t_{\Delta}^{\prime}$. If $e$ is an edge, $\tau \subset e \in c(O) \cap c(\Delta)$ and $\tau$ is not maximal (impossible). Thus the adjacency graph of $L_{\tau}^{\Delta} \cup L_{\tau}^{O}$ is not connected: $\tau$ is not $\partial(O \cup\{\Delta\})$-good.

If $\tau$ is an edge, $g_{\tau}$ is a cycle of adjacent tetrahedra $\Delta_{0} \Delta_{1} \cdots \Delta_{n} \Delta_{0}$ in $T_{\tau}^{\infty}(n \geq 2)$ where $\Delta_{0}=\Delta$. We have $\Delta_{1} \notin O$ and $\Delta_{n} \notin O$ (otherwise there is $i \in\{1, n\}$ such that $\tau \subset \Delta_{i} \cap \Delta \in c(O) \cap c(\Delta)$ and $\tau$ is not maximal). Since $O_{\tau}$ is included in this cycle and $\Delta \notin O_{\tau} \neq \emptyset$, there are (at least) four pairs $\left(\Delta_{i}, \Delta_{(i+1)} \bmod (n+1)\right.$ such that one tetrahedron is in $O \cup\{\Delta\}$ and the other one is not. Thus, four triangles in $\partial(O \cup\{\Delta\})$ include $\tau: \tau$ is not $\partial(O \cup\{\Delta\})$-good.

Lemma 6 If $|\partial O|$ is a 2-manifold and $c(O) \cap c(\Delta)$ is a $2 D$ pure simplicial complex, $|\partial(O \cup\{\Delta\})|$ is a 2 manifold.

Proof For cases $f \in\{0,4\}$, we choose an arbitrary vertex or edge $\tau$ in $c(\partial(O \cup\{\Delta\}))$, examine the triangles in $(\partial(O \cup\{\Delta\}))_{\tau}$, show that $\tau$ is $\partial(O \cup\{\Delta\})$-good, and conclude using Theorem 1.

If $f=0, \partial \Delta \cap \partial O=\emptyset$. Lemma 2 implies $c(\partial O) \cap$ $c(\partial \Delta)=\emptyset$ and Lemma 3 implies $\partial(O \cup\{\Delta\})=\partial O \cup$

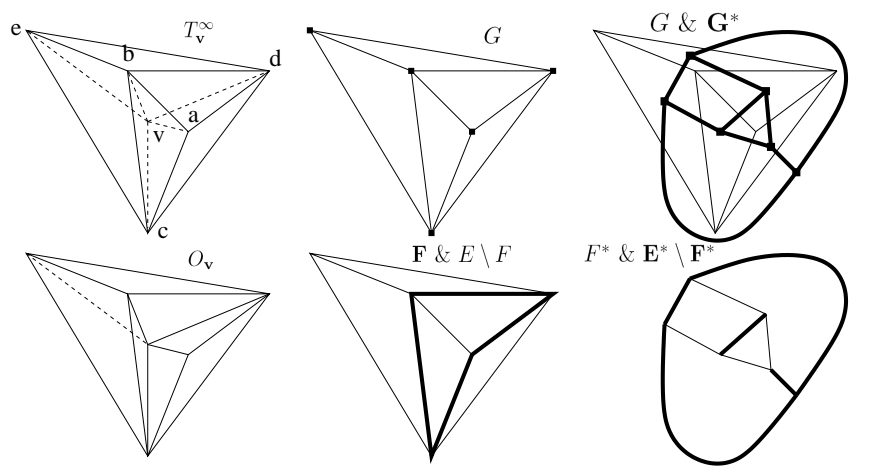

Fig. 4 Notations used in the proof of Theorem 3. Here $\partial T_{\mathbf{v}}^{\infty}=\{\mathbf{a b c}, \mathbf{a c d}, \mathbf{a d b}, \mathbf{e b c}, \mathbf{e c d}, \mathbf{e d b}\}$ (e and $\mathbf{a}$ are in opposite sides of $\mathbf{b c d}$ ) and $O_{\mathbf{v}}=T_{\mathbf{v}}^{\infty} \backslash\{\mathbf{v a b c}, \mathbf{v a d b}\}$. The $G$ edges are in $E$, the $G^{*}$ edges are in $E^{*}, F \subset E, F^{*} \subset E^{*}$. Bold edges are drawn if and only if their names are in bold font (e.g. on the right: $E^{*} \backslash F^{*}$ is bold, $F^{*}$ is not).

$\partial \Delta$. Thus $(\partial(O \cup\{\Delta\}))_{\tau}=(\partial O)_{\tau}$ or $(\partial(O \cup\{\Delta\}))_{\tau}=$ $(\partial \Delta)_{\tau}$. Since $|\partial O|$ and $|\partial \Delta|$ are 2-manifolds, $\tau$ is $\partial O$ good (case 1 ) or $\partial \Delta$-good (case 2 ) by Theorem 1 . We see that $\tau$ is $\partial(O \cup\{\Delta\})$-good in both cases.

If $f=4, \partial \Delta \subseteq \partial O$ and $\partial(O \cup\{\Delta\})=\partial O \backslash \partial \Delta$ (Lemma 3). Assume (reductio ad absurdum) that $\tau \in$ $c(\partial \Delta)$. There is a vertex $\mathbf{v}$ in $c(\partial \Delta) \cap c(\partial O \backslash \partial \Delta)$. Since $|\partial O|$ is a 2-manifold and $\mathbf{v} \in c(\partial O)$, the $\mathbf{v}$-opposite edges in the triangles of $\partial O$ form a cycle (Corollary 2). Since $|\partial \Delta|$ is a 2-manifold and $\mathbf{v} \in c(\partial \Delta)$, the $\mathbf{v}$-opposite edges in the triangles of $\partial \Delta$ form a cycle (Corollary 2 ). Now the second cycle is strictly included in the first one (impossible). We see that $\tau$ is not in a triangle of $\partial \Delta$ and $(\partial(O \cup\{\Delta\}))_{\tau}=(\partial O)_{\tau}$. Since $|\partial O|$ is a 2-manifold, $\tau$ is $\partial O$-good (Theorem 1$)$. Thus $\tau$ is $\partial(O \cup\{\Delta\})$-good.

Assume $f \in\{1,2,3\}$. Since $|\partial O| \cap|\partial \Delta|=|\partial O \cap \partial \Delta|$ (Lemma 2), $|\partial O| \cap|\partial \Delta|$ is homeomorphic to a closed 2-ball. Thus $|\partial(O \cup\{\Delta\})|$ is a 2-manifold since it is a connected sum [12] of two 2-manifolds $|\partial \Delta|$ and $|\partial O|$. An alternative proof is the following: $|\partial(O \cup\{\Delta\})|$ is homeomorphic to $|\partial O|$ (Appendix B) and $|\partial O|$ is a 2manifold, thus $|\partial(O \cup\{\Delta\})|$ is also a 2-manifold.

\section{Proof of the "Tetrahedron-based Test"}

Let $\mathbf{v}$ be a vertex in $c(\partial O)$. Let $G$ be the graph with the vertices $V$ and the edges $E$ in $c\left(\partial T_{\mathbf{v}}^{\infty}\right)$. Let $V^{*}$ be the triangles in $c\left(\partial T_{\mathbf{v}}^{\infty}\right)$, i.e. $V^{*}=\partial T_{\mathbf{v}}^{\infty}$. Let $G^{*}$ be the adjacency graph of the triangles in $V^{*}$. Let $E^{*}$ be the $G^{*}$ edges, i.e. $e^{*} \in E^{*}$ is an edge between triangles $\left\{t_{j}, t_{k}\right\} \subseteq V^{*}$ if $t_{j} \cap t_{k}$ is an edge $e \in E$. The top of Fig. 4 shows $G$ and $G^{*}$ if $T_{\mathbf{v}}^{\infty}$ is a set of 6 tetrahedra.

Here we need an additional definition. A drawing of graph $G=(V, E)$ is a function $\varphi$ defined on $V \cup E$ such that $V$ and $\varphi(V) \subseteq \mathbb{R}^{2}$ are bijective, $\varphi(\mathbf{a b})$ is a non self- 
intersecting curve in $\mathbb{R}^{2}$ whose endpoints are $\varphi(\mathbf{a})$ and $\varphi(\mathbf{b})$ if $\mathbf{a b} \in E$. Furthermore, two such curves cannot intersect except at their endpoints.

The proof of Theorem 3 has six steps:

1. show that $e \mapsto e^{*}$ is a bijection between $E$ and $E^{*}$.

2. find a $G$ drawing using a realization of $c\left(T_{\mathbf{v}}^{\infty}\right)$ in $\mathbb{R}^{3}$.

3. show that $G^{*}$ has a drawing which is dual [3] to $G$ drawing (Fig. 4 shows one example for $G$ and $G^{*}$ ).

4. express Theorem 3 in terms of $G^{*}$ and a minimal cut of $G^{*}$.

5. express Corollary 2 in terms of $G$ and a cycle of $G$.

6. show that Theorem 3 and Corollary 2 are equivalent through duality [3] between cycles of $G$ and minimal cuts of $G^{*}$.

Step 1. We have $\partial T_{\mathbf{v}}^{\infty}=\left\{\mathbf{a b c}, \mathbf{a b c v} \in T_{\mathbf{v}}^{\infty}\right\}$. Therefore $g_{\mathbf{v}}$ (the adjacency graph of tetrahedra in $T_{\mathbf{v}}^{\infty}$ ) and $G^{*}$ (the adjacency graph of triangles in $\partial T_{\mathbf{v}}^{\infty}$ ) are the same. Since every triangle abv is included in exactly two tetrahedra in $T^{\infty}$, every edge $\mathbf{a b} \in E$ is included in exactly two triangles in $V^{*}$. The function $e \mapsto e^{*}$ is thus well defined and bijective between $E$ and $E^{*}$.

Step 2. First we need a realization $K$ of $c\left(T_{\mathbf{v}}^{\infty}\right)$ in $\mathbb{R}^{3}$. If $\mathbf{v} \notin c(\partial T), T_{\mathbf{v}}^{\infty}$ does not contain infinite tetrahedra and $c\left(T_{\mathbf{v}}^{\infty}\right)$ is a simplicial complex. We use $K=$ $c\left(T_{\mathbf{v}}^{\infty}\right)$. If $\mathbf{v} \in c(\partial T), T_{\mathbf{v}}^{\infty}$ contains infinite tetrahedra. In this case, another $K$ is required.

Lemma $7 c\left(T_{\mathbf{v}}^{\infty}\right)$ has realization $K$ in $\mathbb{R}^{3}$ if $\mathbf{v} \in c(\partial T)$.

Proof Note that $c\left(T_{\mathbf{v}}^{\infty}\right)$ is the union of two abstract simplicial complexes: $c\left(T_{\mathbf{v}}\right)$ and the closure of the $\mathbf{v} \mathbf{v}_{\infty^{-}}$ incident tetrahedra in $c\left(T^{\infty}\right)$. The former is also a simplicial complex in $\mathbb{R}^{3}$. The idea of the proof is the following. We find $\mathbf{w} \in \mathbb{R}^{3}$ such that the latter has realization $K^{\prime}$ in $\mathbb{R}^{3}$ by replacing $\mathbf{v}_{\infty}$ by $\mathbf{w}$, and such that $K^{\prime} \cup c\left(T_{\mathbf{v}}\right)$ is a simplicial complex. We obtain $K=K^{\prime} \cup c\left(T_{\mathbf{v}}\right)$.

Let $J=\{\mathbf{v a b}, \mathbf{v a b} \in \partial T\}$ and $\mathbf{w} \in \mathbb{R}^{3}$. Assume

1. if $\mathbf{v a b} \in J,\{\mathbf{w}, \mathbf{v}, \mathbf{a}, \mathbf{b}\}$ are in general position.

Let $\mathbf{w} * J=\{\mathbf{w} \sigma, \sigma \in c(J)\}$ where $\mathbf{w} \sigma$ is the geometric simplex whose vertex set is that of $\sigma$ plus w. Assume

2. if $\left\{\mathbf{w} \sigma, \mathbf{w} \sigma^{\prime}\right\} \subseteq \mathbf{w} * J,(\mathbf{w} \sigma) \cap\left(\mathbf{w} \sigma^{\prime}\right)$ is empty or a face of $\mathbf{w} \sigma$ and $\mathbf{w} \sigma^{\prime}$.

Thus $K^{\prime}=\{\mathbf{w}\} \cup c(J) \cup(\mathbf{w} * J)$ is a simplicial complex: it is the cone [5] on $c(J)$ with vertex w. Assume

3. if $\sigma_{1} \in c\left(T_{\mathbf{v}}\right)$ and $\sigma_{2} \in K^{\prime}, \sigma_{1} \cap \sigma_{2}$ is empty or a face of $\sigma_{1}$ and $\sigma_{2}$.

Thus $K=K^{\prime} \cup c\left(T_{\mathbf{v}}\right)$ is a simplicial complex.

Now we choose $\mathbf{w}$ to meet (1), (2) and (3). Every triangle $t$ in $\partial T$ is included in a plane $\pi_{t}$ which defines two open half-spaces $H_{t}$ and $H_{t}^{\prime}$. The open convex hull
$C$ of Delaunay $T$ meets $C=\cap_{t \in \partial T} H_{t}$. Let $U=\cap_{t \in J} H_{t}$ and $U^{\prime}=\cap_{t \in J} H_{t}^{\prime}$. $U$ and $U^{\prime}$ are opposite half-cones with apex $\mathbf{v}$. Since $\emptyset \neq C \subseteq U$, we have $U^{\prime} \neq \emptyset$ and get $\mathbf{w} \in U^{\prime}$.

We have (1) since $\mathbf{w} \notin \cup_{t \in J} \pi_{t} ;(2)$ is a consequence of $(\mathbf{w} \sigma) \cap\left(\mathbf{w} \sigma^{\prime}\right)=\mathbf{w}\left(\sigma \cap \sigma^{\prime}\right)$. We have $\mathbf{w}\left(\sigma \cap \sigma^{\prime}\right) \subseteq(\mathbf{w} \sigma) \cap$ $\left(\mathbf{w} \sigma^{\prime}\right)$. Conversely, let $\mathbf{x}$ be a point in $(\mathbf{w} \sigma) \cap\left(\mathbf{w} \sigma^{\prime}\right)$ and show $\mathbf{x} \in \mathbf{w}\left(\sigma \cap \sigma^{\prime}\right)$. Let $\mathbf{a}$ be a point in $\sigma$ and $\mathbf{b}$ be a point in $\sigma^{\prime}$ such that $\mathbf{x} \in \mathbf{w a} \cap \mathbf{w b}$. Assume (reductio ad absurdum) that $\mathbf{a} \neq \mathbf{b}$. Since $\mathbf{w}, \mathbf{x}, \mathbf{a}, \mathbf{b}$ are collinear, $\mathbf{a} \in \mathbf{w b}$. Let $t$ be a triangle in $J$ such that $\mathbf{b} \in t$. Thus $\mathbf{w} \in H_{t}^{\prime}$ and $\mathbf{b} \in \pi_{t}$ and $\mathbf{a} \in \mathbf{w} \mathbf{b} \backslash\{\mathbf{b}\}$ imply $\mathbf{a} \in H_{t}^{\prime}$, which contradicts $\mathbf{a} \in|T|$. Since $\mathbf{a}=\mathbf{b}, \mathbf{x} \in \mathbf{w}\left(\sigma \cap \sigma^{\prime}\right)$.

Lastly we show (3). Since $\sigma_{2} \in K^{\prime}=c(\mathbf{w} * J)$, there is a triangle $t$ in $J$ such that $\sigma_{2}$ is a face of $t \mathbf{w}$. Thus $\sigma_{2} \subset \pi_{t} \cup H_{t}^{\prime}$. Furthermore, $\sigma_{1} \subseteq|T| \subset \pi_{t} \cup H_{t}$. Thus $\sigma_{1} \cap \sigma_{2} \subset \pi_{t}$. Since $\sigma_{2} \cap \pi_{t}=\sigma_{2} \cap t$, we have $\sigma_{1} \cap \sigma_{2} \subseteq t$. Assume $\sigma_{1} \cap \sigma_{2} \neq \emptyset$. Note that $t \in J \subseteq c\left(T_{\mathbf{v}}\right) \cap K^{\prime}$. This implies $\sigma_{i} \cap t \in c(J)$ and $\sigma_{i} \cap t$ is a face of $\sigma_{i}$ if $i \in\{1,2\}$. Since $c(J)$ is a simplicial complex, $\sigma_{1} \cap \sigma_{2}=$ $\left(\sigma_{1} \cap t\right) \cap\left(\sigma_{2} \cap t\right)$ is a face of $\sigma_{1} \cap t$ and $\sigma_{2} \cap t$. Now we see that $\sigma_{1} \cap \sigma_{2}$ is empty or a face of $\sigma_{1}$ and $\sigma_{2}$.

Then we would like a drawing $\varphi$ of $G$. Since $c\left(T_{\mathbf{v}}^{\infty}\right)$ has realization $K$ and $G$ is an abstract simplicial complex included in $c\left(\partial T_{\mathbf{v}}^{\infty}\right), G$ has a realization included in $c(\partial K)$. Now $|G|$ is well defined and $|G| \subset|\partial K|$. We use the following lemma to obtain $\varphi$.

Lemma 8 Let $\mathbf{p}$ be a point in $|\partial K| \backslash|G|$. There is a homeomorphism $\varphi$ such that $\varphi(|\partial K| \backslash\{\mathbf{p}\})=\mathbb{R}^{2}$.

Proof The proof has three steps: show that there exists a sphere $S$ included in $|K|$ whose center is $\mathbf{v}$, find homeomorphism $\varphi_{1}$ such that $\varphi_{1}(|\partial K|)=S$, define $\varphi=\varphi_{2} \circ \varphi_{1}$ where $\varphi_{2}$ is homeomorphism such that $\varphi_{2}\left(S \backslash \varphi_{1}(\mathbf{p})\right)=\mathbb{R}^{2}$.

For the first step, we need the following assertion whose technical proof is in Appendix C: if $K$ is a 3D pure simplicial complex in $\mathbb{R}^{3}$ and $\mathbf{x} \in|K| \backslash|\partial K|$, there exists a 3-ball $B$ centered at $\mathbf{x}$ such that $B \subseteq|K|$. Since $\partial T_{\mathbf{v}}^{\infty}=\left\{\mathbf{a b c}, \mathbf{a b c v} \in T_{\mathbf{v}}^{\infty}\right\}, \mathbf{v} \notin|\partial K|$. Now we take $\mathbf{x}=\mathbf{v}$ and obtain a sphere $S$ centered at $\mathbf{v}$ with radius $\epsilon>0$ such that $S \subseteq|K|$.

Half-line $l$ in $\mathbb{R}^{3}$ started at $\mathbf{v}$ intersects $S$ at a single point $\mathbf{p}_{2}$. Let abcv be a tetrahedron in $K$ such that $\mathbf{p}_{2} \in \mathbf{a b c v}$. Thus $l$ intersects $|\partial K|$ at a single point $\mathbf{p}_{1}$ such that $\mathbf{p}_{1} \in \mathbf{a b c}$. Let $\varphi_{1}$ be the function such that $\varphi_{1}\left(\mathbf{p}_{1}\right)=\mathbf{p}_{2}$. We have $\varphi_{1}\left(\mathbf{p}_{1}\right)=\mathbf{v}+\epsilon \frac{\mathbf{p}_{1}-\mathbf{v}}{\left\|\mathbf{p}_{1}-\mathbf{v}\right\|}$ and $\varphi_{1}$ is a homeomorphism ${ }^{1}$ between $|\partial K|$ and $S$. Since $\mathbb{R}^{2}$ and a sphere minus a point are homeomorphic, there is a homeomorphism $\varphi_{2}$ such that $\left.\varphi_{2}\left(S \backslash \varphi_{1}(\mathbf{p})\right)\right)=\mathbb{R}^{2}$.

\footnotetext{
1 Addendum: this is detailed in Appendix D.
} 
Step 3. In this step, we first construct a drawing of $G^{*}$ from the images by $\varphi$ of curves $C_{i}$ included in $|\partial K|$, then we check that this drawing and that of $G$ (defined from the images by $\varphi$ of edges $\left.e_{i} \subset|\partial K|\right)$ are dual [3].

Let $\mathbf{v}_{j}^{*}=(\mathbf{a}+\mathbf{b}+\mathbf{c}) / 3$ for every triangle $t_{j}=\mathbf{a b c} \in$ $\partial K$. Let $\mathbf{m}_{i}=(\mathbf{a}+\mathbf{b}) / 2$ for every edge $e_{i}=\mathbf{a b} \in$ $c(\partial K)$. Since $e_{i} \mapsto e_{i}^{*}$ is bijective (step 1 ), there are exactly two triangles $t_{j}$ and $t_{k}$ of $V^{*}$ such that $e_{i}=t_{j} \cap$ $t_{k}$. Let $C_{i}$ be the union of line segments $\mathbf{v}_{j}^{*} \mathbf{m}_{i}$ and $\mathbf{m}_{i} \mathbf{v}_{k}^{*}$. We have $C_{i} \subseteq t_{j} \cup t_{k} \subseteq|\partial K|$. Let $\mathbf{p}$ be a point in $|\partial K| \backslash$ $\left(\cup_{i} C_{i} \cup|G|\right)$. We use Lemma 8 and $\mathbf{p}$ to obtain $\varphi(\varphi$ is a drawing of $G)$. Let $\varphi^{*}$ be the function defined on $V^{*} \cup E^{*}$ such that $\varphi^{*}\left(t_{j}\right)=\varphi\left(\mathbf{v}_{j}^{*}\right)$ if $t_{j} \in V^{*}$ and $\varphi^{*}\left(e_{i}^{*}\right)=$ $\varphi\left(C_{i}\right)$ if $e_{i}^{*} \in E^{*}$. Now $\varphi^{*}$ meets the requirements of a drawing of $G^{*}$ (note that curves $\varphi^{*}\left(e_{i}^{*}\right)$ and $\varphi^{*}\left(e_{j}^{*}\right)$ cannot intersect except at their endpoints, as curves $C_{i}$ and $C_{j}$ do).

We check that the drawings of $G$ and $G^{*}$ are dual according to Sec. 4.6 of [3]: $G$ is connected (Sec. 2) and has a drawing, there are bijections between $G$ face $f_{j}=$ $\varphi\left(t_{j} \backslash\left|\partial t_{j}\right|\right)$ and $G^{*}$ vertex $\varphi^{*}\left(t_{j}\right)$ such that $\varphi^{*}\left(t_{j}\right) \in f_{j}$, between $G$ edge $\varphi\left(e_{i}\right)$ and $G^{*}$ edge $\varphi^{*}\left(e_{i}^{*}\right)$ such that $\varphi\left(e_{i}\right) \cap \varphi^{*}\left(G^{*}\right)=\varphi\left(e_{i}\right) \cap \varphi^{*}\left(e_{i}^{*}\right)=\varphi(G) \cap \varphi^{*}\left(e_{i}^{*}\right)=$ $\varphi\left(\mathbf{m}_{i}\right)$. This triple equality means that $\varphi\left(e_{i}\right)$ only intersects $\varphi^{*}\left(G^{*}\right)$ at a single point in $\varphi^{*}\left(e_{i}^{*}\right)$, and similarly for the intersection between $\varphi^{*}\left(e_{i}^{*}\right)$ and $\varphi(G)$. This is illustrated at the top right corner of Fig. 4.

Step 4. Since $\mathbf{v} \in c(\partial O), O_{\mathbf{v}} \neq \emptyset$ and $O_{\mathbf{v}}^{c} \neq \emptyset$. The partition $\left\{O_{\mathbf{v}}, O_{\mathbf{v}}^{c}\right\}$ of $T_{\mathbf{v}}^{\infty}$ defines a partition $\left\{V_{O}^{*}, V_{O^{c}}^{*}\right\}$ of $V^{*}: t \in V_{O}^{*}$ iff $t$ is the $\mathbf{v}$-opposite triangle of a tetrahedron in $O_{\mathbf{v}}$, and similarly for $V_{O^{c}}^{*}$ and $O_{\mathbf{v}}^{c}$. Let $F^{*}$ be the edges of graph $G^{*}$ having a vertex in $V_{O^{c}}^{*}$ and a vertex in $V_{O}^{*}$. Since $g_{\mathbf{v}}=G^{*}=\left(V^{*}, E^{*}\right)$ (step 1 ), we have $g_{\mathbf{v}}^{O}=\left(V^{*}, E^{*} \backslash F^{*}\right)$. Fig. 4 shows one example for $E^{*}$ and $F^{*}$ where $O_{\mathbf{v}}^{c}$ has two tetrahedra. According to Theorem 3, v is regular in $|\partial O|$ iff $g_{\mathbf{v}}^{O}$ exactly has two connected components, i.e. iff $F^{*}$ is a minimal cut in $G^{*}$.

Step 5. Let $F$ be the edges of graph $G$ included in a $V_{O^{c}}^{*}$ triangle and in a $V_{O}^{*}$ triangle. Fig. 4 shows one example for $F$ where $V_{O^{c}}^{*}$ has two triangles. We have $f \in F$ iff there are vertices $\mathbf{a}, \mathbf{b}, \mathbf{c}, \mathbf{c}^{\prime}$ such that $f=\mathbf{a b}$ and $\mathbf{a b v c} \in O$ and $\mathbf{a b v c}^{\prime} \in O^{c}$, i.e. iff $f$ is a v-opposite edge in a triangle of $\partial O$. According to Corollary 2, $\mathbf{v}$ is regular in $|\partial O|$ iff $F$ is a cycle in $G$.

Step 6. Note that every edge in $F^{*}$ is dual to an edge in $F$ by bijection $e_{i} \mapsto e_{i}^{*}$ (step 1 ). Since $G$ is connected (Sec. 2) and has a drawing (step 3), and thanks to the duality between $G$ and $G^{*}$ (step 3 ) and between $F$ and $F^{*}$, we use Proposition 4.6.1 in [3]: $F$ is a cycle in $G$ iff $F^{*}$ is a minimal cut in $G^{*}$. Now we see that Theorem 3 and Corollary 2 are equivalent.

\section{Conclusion}

Two other theoretical topics related to surface reconstruction based on these tests should be investigated. First we do not know if the problem of finding a 2manifold surface maximizing the visibility score in [7] is NP-hard. Second we do not know if there is a growing algorithm whose growing list of tetrahedra can reach every 2-manifold embedded in the 3D Delaunay.

\section{A Proofs of prerequisites for Theorem 4}

We remember that $O \subset T^{\infty}, \partial O \subset c(T)$ and $\Delta \in T \backslash O$.

\section{A.1 Proof of Lemma 1}

Since $c(O)$ and $c(\Delta)$ are abstract simplicial complexes included in abstract simplicial complex $c\left(T^{\infty}\right), c(O) \cap c(\Delta)$ is an abstract simplicial complex included in $c\left(T^{\infty}\right)$. Since $\Delta \in T, c(\Delta)$ is also a simplicial complex and $c(O) \cap c(\Delta)$ has a realization included in simplicial complex $c(\Delta)$. We use the same notation for $c(O) \cap c(\Delta)$ and its realization in $\mathbb{R}^{3}$.

We have $c(\partial O) \cap c(\partial \Delta) \subseteq c(O) \cap c(\Delta)$ since $\partial O \subset c(O)$ and $\partial \Delta \subset c(\Delta)$. Conversely, let $\sigma$ be an arbitrarily chosen simplex from $c(O) \cap c(\Delta)$ and show $\sigma \in c(\partial O) \cap c(\partial \Delta)$. First we study the case where $\sigma$ is a triangle. Since $\Delta \notin O, \sigma$ is included in $\Delta$ and in another tetrahedron which is in $O$. Thus $\sigma \in \partial \Delta \cap \partial O \subset c(\partial O) \cap c(\partial \Delta)$. Now we study the case where $\sigma$ is a vertex or an edge. Since $\sigma \in c(O), O_{\sigma} \neq \emptyset$. Furthermore $g_{\sigma}$ is a connected graph. Therefore, there is a series $\Delta_{0} \Delta_{1} \cdots \Delta_{n}$ of adjacent tetrahedra where $\Delta_{0}=\Delta$, $\Delta_{n} \in O_{\sigma}$ and $\forall i \in\{1, \cdots n-1\}, \Delta_{i} \in T_{\sigma}^{\infty} \backslash(O \cup\{\Delta\})$. Note that $\Delta \notin O$ implies $n>0$ and triangle $\Delta_{0} \cap \Delta_{1} \in \partial \Delta$ and triangle $\Delta_{n-1} \cap \Delta_{n} \in \partial O$. Since $\sigma$ is included in all the $\Delta_{i}$ above, $\sigma \subseteq \Delta_{0} \cap \Delta_{1}$ and $\sigma \subseteq \Delta_{n-1} \cap \Delta_{n}$. Thus $\sigma \in c(\partial \Delta)$ and $\sigma \in c(\partial O)$.

\section{A.2 Proof of Lemma 2}

Note that $|\partial O|$ is well defined since $\partial O \subset c(T)$. Point $\mathbf{p} \in$ $|\partial \Delta| \cap|\partial O|$ iff there are geometric triangles $t$ and $t^{\prime}$ such that $\mathbf{p} \in t \in \partial \Delta$ and $\mathbf{p} \in t^{\prime} \in \partial O$. If $\mathbf{p} \in|\partial \Delta| \cap|\partial O|$, $\mathbf{p} \in t \cap t^{\prime} \in c(\partial \Delta) \cap c(\partial O)$ and we obtain $\mathbf{p} \in|c(\partial \Delta) \cap c(\partial O)|$. If $\mathbf{p} \in|c(\partial \Delta) \cap c(\partial O)|$, there is a geometric simplex $\sigma$ such that $\mathbf{p} \in \sigma \in c(\partial \Delta) \cap c(\partial O)$. Thus there are triangles $t$ and $t^{\prime}$ such that $\sigma \subseteq t \cap t^{\prime}$ and $t \in \partial \Delta$ and $t^{\prime} \in \partial O$. We obtain $\mathbf{p} \in|\partial \Delta| \cap|\partial O|$.

Thanks to Lemma 1, $|\partial O| \cap|\partial \Delta|=|c(\partial O) \cap c(\partial \Delta)|=$ $|c(O) \cap c(\Delta)|$ and the triangles of $c(O) \cap c(\Delta)$ are $\partial O \cap \partial \Delta$. Thus $|\partial O| \cap|\partial \Delta|=|\partial O \cap \partial \Delta|$ if $c(O) \cap c(\Delta)$ is $2 \mathrm{D}$ pure.

\section{A.3 Proof of Lemma 3}

Since $\Delta \notin O$, triangle $t$ is in $\partial O \cap \partial \Delta$ iff the two tetrahedra including $t$ are $\Delta$ and another one in $O$. Thus, $t \in(\partial O \cup \partial \Delta) \backslash$ $(\partial O \cap \partial \Delta)$ iff triangle $t$ is a face of exactly one tetrahedron of $O \cup\{\Delta\}$, i.e. iff $t \in \partial(O \cup\{\Delta\})$. 


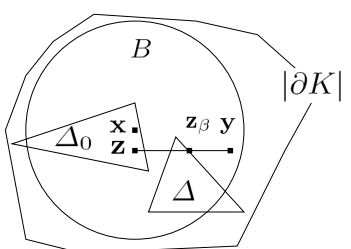

Fig. 5 Notations for the reductio ad absurdum of Lemma 8 (tetrahedra $\Delta_{0}$ and $\Delta$ are represented by triangles). This figure suggests that $\mathbf{y} \in|K|$, which is wrong.

\section{B Alternative proof for Lemma $6(f \in\{1,2,3\})$}

Here we find homeomorphism $\varphi$ such that $\varphi(|\partial O|)=\mid \partial(O \cup$ $\{\Delta\}) \mid . \varphi$ is defined by its values on vertices and linear interpolation on $\partial O$ triangles (or their subdivisions) as follows. For every vertex $\mathbf{v}$ in $c(\partial O)$, we set $\varphi(\mathbf{v})=\mathbf{v}$. We use notation $\Delta=$ abcd.

If $f=1,|\partial O \cap \partial \Delta|=\mathbf{a b c}$, we split abc into triangles abe, bce, cae where $\mathbf{e}=(\mathbf{a}+\mathbf{b}+\mathbf{c}) / 3$, and set $\varphi(\mathbf{e})=\mathbf{d}$. We obtain $\varphi(\mathbf{a b c})=\mathbf{a b d} \cup \mathbf{b c d} \cup \mathbf{c a d}$.

If $f=2,|\partial O \cap \partial \Delta|=\mathbf{a b c} \cup \mathbf{b c d}$, we split bc into edges be and ec where $\mathbf{e}=(\mathbf{b}+\mathbf{c}) / 2$, we split ad into edges ag and gd where $\mathbf{g}=(\mathbf{a}+\mathbf{d}) / 2$, and set $\varphi(\mathbf{e})=\mathbf{g}$. Note that this scheme also splits every $\Delta$ triangle into two other triangles. We obtain $\varphi(\mathbf{a b c} \cup \mathbf{b c d})=\mathbf{a d b} \cup$ adc.

If $f=3,|\partial O \cap \partial \Delta|=\mathbf{d a b} \cup \mathbf{d b c} \cup \mathbf{d} \mathbf{c a}$, we split abc into abe, bce, cae where $\mathbf{e}=(\mathbf{a}+\mathbf{b}+\mathbf{c}) / 3$, and reset $\varphi(\mathbf{d})=\mathbf{e}$. We obtain $\varphi($ dab $\cup$ dbc $\cup$ dca $)=$ abc.

In all cases, $\varphi(|\partial O \cap \partial \Delta|)=|\partial \Delta \backslash \partial O|$ and $\varphi$ is the identity in $|\partial O \backslash \partial \Delta|$. Since $\partial(O \cup\{\Delta\})=(\partial O \backslash \partial \Delta) \cup(\partial \Delta \backslash \partial O)$ (Lemma 3), $\varphi(|\partial O|)=|\partial(O \cup\{\Delta\})|$. Lastly, $\varphi$ is a homeomorphism between $|\partial O|$ and $|\partial(O \cup\{\Delta\})|$ since [9] it maps the $|\partial O|$ vertices to distinct points ( $\varphi$ is a simplicial map).

\section{Proof for Lemma 8}

Here we show the following assertion: if $K$ is a 3D pure simplicial complex in $\mathbb{R}^{3}$ and $\mathbf{x} \in|K| \backslash|\partial K|$, there exists a 3-ball $B$ centered at $\mathbf{x}$ such that $B \subseteq|K|$.

First we study a special case: $K=c\left(\left\{\Delta, \Delta^{\prime}\right\}\right)$ where $\Delta$ and $\Delta^{\prime}$ are two tetrahedra sharing a triangle $t$ and $\mathbf{x} \in t \backslash|\partial t|$. Let $h_{0}, h_{0}^{\prime}, h_{1}, h_{2} \cdots h_{6}$ be the half-spaces such that $\Delta=h_{0} \cap$ $h_{1} \cap h_{2} \cap h_{3}$ and $\Delta^{\prime}=h_{0}^{\prime} \cap h_{4} \cap h_{5} \cap h_{6}$ and $h_{0} \cup h_{0}^{\prime}=\mathbb{R}^{3}$. Let $r=\min _{1 \leq i \leq 6} d\left(\mathbf{x}, h_{i}\right)$ where $d\left(\mathbf{x}, h_{i}\right)$ is the Euclidean distance between $\mathbf{x}$ and the border plane of $h_{i}$. We have $0<$ $r$ (otherwise $\Delta$ or $\Delta^{\prime}$ is degenerate). Let $B$ be the 3-ball centered at $\mathbf{x}$ with radius $r$. Since $1 \leq i \leq 6$ implies $B \subseteq h_{i}$, we have $B \cap h_{0} \subseteq \Delta$ and $B \cap h_{0}^{\prime} \subseteq \Delta^{\prime}$. Thus $B \subseteq \Delta \cup \Delta^{\prime}=|K|$.

Then we study the general case. Since $K$ is $3 \mathrm{D}$ pure, there is a tetrahedron $\Delta_{0} \in K$ such that $\mathbf{x} \in \Delta_{0}$. Since $|\partial K|$ is closed (indeed, it is a finite union of geometric simplices), $\mathbb{R}^{3} \backslash|\partial K|$ is open. Thus there is a 3 -ball $B$ centered at $\mathbf{x}$ such that $B \cap|\partial K|=\emptyset$. Now we show that $B \subseteq|K|$.

Assume (reductio ad absurdum) that there is $\mathbf{y} \in B \backslash|K|$. Let $\mathbf{z} \in B \cap \Delta_{0}$ such that line segment zy does not intersect the $K$ edges. Let $\mathbf{z}_{\alpha}=(1-\alpha) \mathbf{z}+\alpha \mathbf{y}$ where $\alpha \in[0,1]$. Let $\beta=\sup _{\mathbf{z}_{\alpha} \in|K|} \alpha$. Since $\mathbf{z}_{0} \in|K|, \beta \in[0,1]$. Since $|K|$ is closed, $\mathbf{z}_{\beta} \in|K|$. There is a tetrahedron $\Delta$ in $K$ such that $\mathbf{z}_{\beta} \in \Delta$. Now $\mathbf{z}_{\beta} \in \Delta$ and $\mathbf{z}_{1} \notin \Delta$ imply that there is $\gamma \in[\beta, 1[$ such that $\mathbf{z}_{\gamma} \in|\partial \Delta|$. Since $|\partial \Delta| \subset|K|, \gamma \leq \beta$. Thus $\gamma=\beta$ and $\mathbf{z}_{\beta}$ is in a triangle $t$ of $\partial \Delta$. Fig. 5 shows $B,|\partial K|, \Delta_{0}, \Delta$, $\mathbf{x}, \mathbf{y}, \mathbf{z}$ and $\mathbf{z}_{\beta}$. Now there are two cases.
If $t \notin \partial K, t=\Delta \cap \Delta^{\prime}$ where $\Delta^{\prime}$ is a tetrahedron in $K \backslash\{\Delta\}$. Since $\mathbf{z}_{\beta}$ is not in the $K$ edges, we use the special case above and obtain a 3 -ball $B^{\prime}$ centered at $\mathbf{z}_{\beta}$ and included in $|K|$. Now there is $\alpha \in] \beta, 1]$ such that $\mathbf{z}_{\alpha} \in B^{\prime} \subseteq|K|$ (impossible).

If $t \in \partial K, \mathbf{z}_{\beta} \in t$ and $B \cap|\partial K|=\emptyset$ imply $\mathbf{z}_{\beta} \notin B$. However, $B$ is convex and $\{\mathbf{z}, \mathbf{y}\} \subset B$ and $\mathbf{z}_{\beta} \in \mathbf{z y}$ imply $\mathbf{z}_{\beta} \in B$ (impossible).

\section{Addendum}

Here we detail the assertion " $\varphi_{1}$ is homeomorphism" in the proof of Lemma 8 .

First we show that $\varphi_{1}$ is injective. Let $\{\mathbf{x}, \mathbf{y}\} \subseteq|\partial K|$ such that $\varphi_{1}(\mathbf{x})=\varphi_{1}(\mathbf{y})$. We use notation $\stackrel{\circ}{\sigma}=\sigma \backslash|\bar{\partial} \sigma|$ if $\sigma$ is a geometric simplex $(\stackrel{\circ}{\sigma}=\sigma$ if $\sigma$ is a vertex $)$. Let simplex $\sigma_{x} \in c(\partial K)$ such that $\mathbf{x} \in \sigma_{x}^{\circ}: \mathbf{x}$ is at a vertex, or in an edge without its 2 end vertices, or in a triangle without its 3 edges. Let triangle $t_{y} \in \partial K$ such that $\mathbf{y} \in t_{y}$. Both simplex $\mathbf{v} \sigma_{x}$ and tetrahedron $\mathbf{v} t_{y}$ are in $K$. Let $\sigma=\mathbf{v} \sigma_{x}$ and $\sigma^{\prime}=\mathbf{v} t_{y}$. Since $\mathbf{x} \in \sigma_{x}^{\circ}$ and $\mathbf{y}$ is in the half-line started at $\mathbf{v}$ that includes $\mathbf{x}, \stackrel{\circ}{\sigma} \cap \sigma^{\prime} \neq \emptyset$. According to Lemma 1 in the supplementary material, $\sigma \subseteq \sigma^{\prime}$. This implies $\sigma_{x} \subseteq t_{y}$, thus $\mathbf{x}=\mathbf{y}$.

Last we show that $\varphi_{1}^{-1}$ is continuous. Let triangle $t \in \partial K$ and point $\mathbf{p}_{2} \in \varphi_{1}(t)$. Since $\varphi_{1}$ is injective, $\varphi_{1}^{-1}\left(\mathbf{p}_{2}\right) \in t$. Thus $\varphi_{1}^{-1}$ maps $\mathbf{p}_{2}$ to the intersection between $t$ and the line including both $\mathbf{v}$ and $\mathbf{p}_{2}$. We see that function $\varphi_{1}^{-1}$ restricted to $\varphi_{1}(t)$ is continuous. Then the pasting lemma implies that $\varphi_{1}^{-1}$ is continuous in the finite union of closed sets $\varphi_{1}(t)$ where $t \in \partial K$, i.e $\varphi_{1}^{-1}$ is continuous in $S$.

\section{References}

1. Boissonnat J.D., Devillers O., Pion S., Teillaud M., Yvinec M.: Triangulations in CGAL. Computational Geometry: Theory and Applications 22(5), 2002

2. Boissonnat J.D.: Geometric structures for threedimensional shape representation. ACM Transactions on Graphics 3(4), 266-286 (1984)

3. Diestel R.: Graph theory. Springer Verlag (graduated texts in mathematics 173) (2010)

4. Gezahegne A.: Surface reconstruction with constrained sculpting. Master of Science Thesis. University of California Davis (2005)

5. Giblin P.: Graphs, surfaces and homology. Cambridge University Press (third edition, 2010)

6. Goodman J., Rourke J.O. (eds): Handbook of discrete and computational geometry, CRC. Press (2004)

7. Lhuillier M., Yu S.: Manifold surface reconstruction of an environment from sparse Structure-from-Motion data. CVIU 117(11), 1628-1644 (2013)

8. Litvinov V., Lhuillier M.: Incremental solid modeling from sparse and omnidirectional structure-from-motion data. BMVC 2013.

9. Sakai K.: Simplicial Homology, a Short Course. Institute of Mathematics, University of Tsukuba (2010)

10. Vegter G.: Computational Topology. In: Goodman J., Rourke J.O. (eds) Handbook of discrete and computational geometry, CRC. Press (2004)

11. Yu S., Lhuillier M.: Incremental reconstruction of manifold surface from sparse visual mapping. 3DIMPVT 2012.

12. Zomorodian A.J.: Topology for computing. Cambridge University Press (2005) 